\section{Poluição veicular e saúde da população: uma revisão sobre o município de São Paulo (SP), Brasil}

\section{Traffic related air pollution and population health: a review about São Paulo (SP), Brazil}

\section{Giovana lara Ferreira Moser de Toledo'}

\section{Adelaide Cássia Nardocci'}

'Departamento de Saúde Ambiental da Faculdade de Saúde Pública da Universidade de São Paulo (USP) - São Paulo (SP), Brasil

Trabalho realizado no Departamento de Saúde Ambiental da Faculdade de Saúde Pública da Universidade de São Paulo (USP) - São Paulo (SP), Brasil.

Correspondência: Adelaide Cassia Nardocci - Avenida Dr. Arnaldo, 715 - Cerqueira César CEP: 01246-904 - São Paulo (SP), Brasil - E-mail: nardocci@usp.br.

Fonte de financiamento: Coordenação de Aperfeiçoamento de Pessoal de Nível Superior

Conflito de interesse: nada a declarar.

\section{Resumo}

Introdução: A poluição do ar é um problema importante para o município de São Paulo, cuja maior fonte são os veículos. Cerca de 11 milhões de habitantes estão expostos a essa poluição. Objetivo: Analisar os estudos realizados sobre a poluição do ar e seus efeitos na saúde da população do município de São Paulo e sobre métodos de avaliação da exposição à poluição relacionada ao tráfego. Métodos: Foi realizado um levantamento bibliográfico com os descritores "air pollution", "São Paulo" e "traffic-related air pollution". Resultados: Foram obtidos vários estudos que verificaram relações entre a poluição do ar no município de São Paulo e problemas respiratórios e cardiovasculares, crescimento fetal, aumento na mortalidade e hospitalizações, particularmente em idosos e crianças. Para estimativa da exposição, a maioria destes estudos considera a distribuição isotrópica dos poluentes para toda a área, o que impede a avaliação da influência do tráfego. Vários métodos têm sido utilizados para avaliação da exposição da população à poluição relacionada ao tráfego, os quais podem ser utilizados isoladamente ou em conjunto. Acredita-se que a associação de modelos para cálculo das concentrações de poluentes a métodos de georreferenciamento seja a abordagem adequada para o município de São Paulo. As vantagens do uso destes métodos são a capacidade de identificar situações e áreas prioritárias, obtenção de informações detalhadas para adoção de medidas ou políticas públicas e a simulação de diferentes cenários. Conclusão: A aplicação destes métodos, em estudos no município de São Paulo, depende da melhoria dos dados de entrada dos modelos, o aprimoramento da rede de monitoramento do ar e de condições meteorológicas e dados do volume de tráfego.

Palavras-chave: poluição do ar; exposição ambiental; saúde pública; efeitos adversos; saúde ambiental. 


\section{Abstract}

Introduction: Air pollution is an important problem for São Paulo city and vehicles are the main source. About 11 million people are exposed to this pollution. Objective: To examine studies realized about air pollution and its effects on health of the population of São Paulo (Brazil) and methods of assessing exposure to pollution related to traffic. Methods: We performed a literature review using the keywords "air pollution", "São Paulo", and traffic-related air pollution. Results: As results were obtained several studies that found relation between air pollution in São Paulo and respiratory and cardiovascular problems, fetal growth, increased mortality and hospitalizations, particularly in children and elderly people. In order to estimate the exposure, most of these studies consider the isotropic distribution of pollutants throughout the area, what prevents the evaluation of and the influence of traffic. Several methods have been used to analyze the air traffic exposure, which can be used isolated or combined. It is believed that the combination of models used to calculate pollutant concentrations to methods of georeferencing is the most appropriate approach for similar studies in São Paulo. The advantages of these methods are the ability to identify priority areas and situations, obtaining detailed information for adoption of public policies or measures, and to simulate different scenarios. Conclusion: The application of these methods in studies at São Paulo depends on the improvement of input data, air quality meteorological monitoring net enhancement and data of traffic volume.

Keywords: air pollution; environmental exposure; public health; adverse effects; environmental health.

\section{Introdução}

Os efeitos da poluição do ar adversos à saúde humana constituem importante tema de Saúde Pública. Até a década de 1980, a poluição do ar em São Paulo era de origem predominantemente industrial. Em 1990, o aumento do controle das emissões industriais e mudanças na ocupação do solo fizeram com que muitas indústrias se transferissem para outras áreas do Estado ${ }^{1,2}$. Atualmente, a poluição emitida pela frota veicular é a principal fonte de poluição atmosférica $^{3}$.

$\mathrm{O}$ número de veículos que circula na Região Metropolitana de São Paulo (RMSP) é crescente e, em 2008, chegou a 9,2 milhões de veículos, representando um quinto da frota nacional. A emissão anual, de origem veicular, é de 1,56 milhão de toneladas de monóxido de carbono (CO), 367 mil toneladas de óxidos de nitrogênio e 62,3 mil toneladas de material particulado, o que corresponde a $90 \%$ da poluição atmosférica emitida em São Paulo ${ }^{3}$.

A idade da frota é outro fator agravante da poluição do ar na cidade. Em 2005, cerca de $58 \%$ da frota era anterior a 1997 , cujos veículos não dispunham de sistemas de controle de poluição e não eram submetidos à manutenção periódica para minimizar a emissão de poluentes.

O município de São Paulo ocupa uma área de $1.523 \mathrm{~km}^{2}$ e tem uma população de quase 11 milhões de habitantes ${ }^{4}$. A malha viária total é de $18.000 \mathrm{~km}$ de ruas e avenidas, muitas com intenso volume de tráfego todos os dias, apesar de medidas de restrições de circulação de veículos em horários de pico, adotadas nos últimos anos na região central. A frota de ônibus para o transporte público chega a 15.000 veículos $^{5}$.

A poluição do ar no município de São Paulo é monitorada pela Companhia de Tecnologia de Saneamento Ambiental (CETESB), por meio de 12 estações fixas de monitoramento que medem as concentrações dos poluentes: monóxido de carbono (CO), dióxido de enxofre $\left(\mathrm{SO}_{2}\right)$, óxidos de nitrogênio, material particulado e ozônio. 
A partir de 1981, iniciou-se o monitoramento automático da qualidade do ar e, em 1999, teve início o monitoramento manual de material particulado fino $\left(\mathrm{PM}_{2,5}\right)$, o que ainda não ocorre de maneira sistemática ${ }^{3}$.

Neste período, muitos estudos encontraram relações entre as concentrações de poluentes atmosféricos no município de São Paulo e agravos à saúde, como doenças respiratórias e cardiovasculares, medidas a partir do número de internações hospitalares, mortalidade e atendimento em serviços de saúde, especialmente em crianças e ido$\operatorname{sos}^{6-9}$. No entanto, apesar de estes estudos mostrarem a poluição do ar como fator de risco para a população no município de São Paulo, ainda existem lacunas importantes no conhecimento da magnitude dos impactos da exposição a estes poluentes na saúde da população.

O objetivo deste artigo foi analisar os estudos sobre a poluição do ar e seus efeitos na saúde da população do município de São Paulo (SP). Além disso, pretendeu-se, com base nos estudos desenvolvidos internacionalmente, discutir novos métodos de avaliação da exposição à poluição relacionada ao tráfego.

\section{Métodos}

Para o levantamento dos estudos sobre os efeitos à saúde da poluição do ar no município de São Paulo, foram utilizados como descritores os termos "air pollution" e "São Paulo", de 1998 a 2009.

A partir da leitura dos resumos, foram descartados os trabalhos não relacionados diretamente ao tema, como os que envolviam biomarcadores, estudos específicos de variáveis climáticas e estudos com outros contaminantes de solo e água.

Para o levantamento dos métodos empregados na avaliação de exposição à poluição relacionada ao tráfego, foi utilizado o descritor "traffic-related air pollution" e selecionados os trabalhos publicados a partir de 1998. Da mesma forma, foram descartados artigos que abordavam temas como estudos laboratoriais, poluição indoor, biomarcadores e ainda estudos envolvendo a exposição ao ruído ou outros fatores ambientais não relacionados à poluição do ar. Para exemplificar os métodos de avaliação da exposição encontrados, foram citadas as publicações mais recentes de cada método.

Após a obtenção dos artigos completos, foi executada a fase de análise, que consistiu em agrupar as informações sobre os métodos de avaliação da exposição à poluição do ar relacionada ao tráfego.

\section{Resultados}

\section{Poluição do ar e efeitos à saúde na cidade de São Paulo}

Foram encontrados 144 artigos, distribuídos nas seguintes bases: CAB (59), LILACS (42) e PUBMED (43). Foram descartados os artigos não relacionados ao tema e 28 destes foram utilizados nesta etapa da revisão. Muitos estudos encontraram associação entre as concentrações de poluentes atmosféricos e efeitos à saúde das pessoas no município de São Paulo, a partir de dados de mortalidade, internação hospitalar e atendimentos ambulatoriais por doenças respiratórias e cardiovasculares e, ainda, de mortalidade intrauterina e perinatal.

Estudo com idosos encontrou que um aumento de $3-4 \%$ da taxa de mortalidade diária por doenças cardiovasculares estava associado ao aumento de material particulado com diâmetro até $10 \mu \mathrm{m}\left(\mathrm{PM}_{10}\right)$ e $\mathrm{SO}_{2}$. Para doenças respiratórias, o aumento na mortalidade diária foi maior (6\%). As mortes por doenças cardiovasculares também estavam associadas aos níveis de $\mathrm{CO}(4 \%)^{7}$.

Foi verificado que um aumento de $10 \mu \mathrm{g} / \mathrm{m}^{3} \mathrm{de} \mathrm{SO}_{2}$ resultava em um aumento de $2,4 \%$ na mortalidade diária de idosos, com um intervalo de tempo de 3 dias entre $o$ aumento da poluição e o aumento na mortalidade $^{10}$. Também verificou-se que um incremento de $10 \mu \mathrm{g} / \mathrm{m}^{3}$ de $\mathrm{PM}_{10}$ resultou em um aumento de 1,4 a $14,2 \%$ na chance de morte por problemas respiratórios e, 
ainda, que as maiores taxas de mortalidade foram observadas em famílias com piores condições socioeconômicas ${ }^{11}$.

Em outro estudo, foi observada associação entre mortalidade em idosos por doenças respiratórias e cardiovasculares para os poluentes: $\mathrm{PM}_{10}, \mathrm{SO}_{2}$ e $\mathrm{CO}^{12}$. Incrementos de $10 \mu \mathrm{g} / \mathrm{m}^{3}$ nos níveis de poluentes e de 1 ppm nos níveis de CO resultaram em um aumento percentual na mortalidade por doenças do aparelho circulatório de $0,3,1,7$ e 4,9\% e por doenças respiratórias de $0,9,13,7$ e 5,3\% para $\mathrm{PM}_{10}, \mathrm{CO}$ e $\mathrm{SO}_{2}$, respectivamente.

No caso de crianças, foi encontrada associação entre a mortalidade das crianças menores de 5 anos e os poluentes $\mathrm{CO}$, $\mathrm{SO}_{2}$ e $\mathrm{PM}_{10}$, sendo a proporção de mortes atribuídas a estes poluentes de 15, 13 e 7\%, respectivamente ${ }^{13}$.

Estudo envolvendo várias cidades da América Latina concluiu que o $\mathrm{PM}_{10}$ tem um importante papel nos efeitos a curto e médio termo na mortalidade, mas que na América Latina as associações não diferem conforme o nível educacional ${ }^{14}$.

A associação entre as concentrações diárias de dióxido de nitrogênio $\left(\mathrm{NO}_{2}\right), \mathrm{SO}_{2}, \mathrm{CO}$, ozônio $\left(\mathrm{O}_{3}\right), \mathrm{PM}_{10}$ e a mortalidade intrauterina apresentou forte associação para o $\mathrm{NO}_{2}$ e associação moderada para o $\mathrm{SO}_{2}$ e CO. Foi encontrada forte associação quando todos os poluentes foram considerados ${ }^{15}$.

Um estudo caso-controle recente encontrou associação entre a mortalidade perinatal e a exposição à poluição relacionada ao tráfego. Neste caso, a exposição foi avaliada pela distância da via, multiplicada por um fator de peso relacionado à densidade de tráfego da via ${ }^{16}$.

Vários outros estudos utilizaram como desfecho as internações hospitalares. O aumento percentual de internações de idosos por doenças respiratórias associados ao incremento de $1 \mathrm{ppm}$ de $\mathrm{CO}$ e $10 \mu \mathrm{g} / \mathrm{m}^{3}$ de $\mathrm{PM}_{10}$ e $\mathrm{SO}_{2}$ foi de 3,2, 1,9 e 10,8\%, respectivamente ${ }^{12}$. Para crianças, o aumento percentual foi de $6,7 \%$ para $\mathrm{PM}_{10}$ e $\mathrm{SO}_{2}$ e 1,7\% para $\mathrm{CO}$.

Para crianças menores de 13 anos, os poluentes $\mathrm{PM}_{10} \mathrm{eO}_{3}$ apresentaram associação mais forte com admissão hospitalar por doenças respiratórias e um nível médio de $70 \mu \mathrm{g} / \mathrm{m}^{3}$ estava associado a um aumento de $12 \%$ no número de admissões hospitalares, com um período de 1 a 7 dias entre o aumento da poluição e a verificação do seu efeito na saúde das pessoas ${ }^{17}$.

Em outro estudo, verificou-se um aumento do número de internações de crianças por doenças respiratórias e pneumonia devido ao aumento nas concentrações de $\mathrm{O}_{3}(5-8 \%), \mathrm{NO}_{2}(9 \%)$ e $\mathrm{PM}_{10}(9 \%)^{18}$.

Alta prevalência de doenças respiratórias em crianças de 11 a 13 anos foi observada em áreas muito poluídas por $\mathrm{SO}_{2}$ e $\mathrm{PM}_{10}{ }_{10}^{19}$. Este estudo mostrou ainda que a prevalência de doenças respiratórias aumentou em São Paulo de 1986 a 1996, embora os níveis médios de poluição tenham ficado abaixo dos padrões de qualidade do ar. $\mathrm{O}$ estudo destaca ainda que os efeitos de medidas de controle adotadas no período foram neutralizados pelo aumento da frota de veículos.

Os efeitos das concentrações de $\mathrm{O}_{3^{\prime}}, \mathrm{SO}_{2^{\prime}}$ $\mathrm{PM}_{10}, \mathrm{CO}$ e $\mathrm{NO}_{2}$ em vários grupos etários de crianças foram estudados e observou-se que as crianças menores de 2 anos foram as mais suscetíveis, sendo observado um aumento de até $9,4 \%$ nas admissões hospitalares por causas respiratórias, em função do aumento das concentrações de $\mathrm{PM}_{10}$. $\mathrm{O}$ segundo grupo mais afetado foi o de jovens de 14-19 anos $^{8}$. Também foi observado que a admissão hospitalar por doença respiratória em menores de 15 anos estava fortemente associada ao aumento de $\mathrm{PM}_{10}{ }_{10}^{19}$.

Um estudo desenvolvido em várias cidades brasileiras, inclusive em São Paulo, sugere uma associação entre a alta exposição a poluentes fotoquímicos e alta prevalência de sintomas de asma, rinite e eczema atópico ${ }^{20}$. Em outro estudo, os poluentes $\mathrm{O}_{3} \mathrm{e}$ $\mathrm{SO}_{2}$ estavam associados a um acréscimo no número de atendimentos por pneumonia e gripe em idosos, mas estes poluentes não possuíam efeitos independentes ${ }^{9}$.

Verificou-se que todos os poluentes, mas especialmente o $\mathrm{CO}$, estavam associados a um aumento da procura de serviços 
de emergência por casos de angina e infarto agudo do miocárdio ${ }^{21}$. Trabalho mais recente identificou que os efeitos da poluição do ar na arritmia são predominantemente agudos e iniciam-se em concentrações abaixo dos padrões atuais de qualidade do $\operatorname{ar}^{22}$.

Estudo sobre os níveis de $\mathrm{SO}_{2}, \mathrm{CO}, \mathrm{PM}_{10}$, $\mathrm{O}_{3}$ e $\mathrm{NO}_{2}$ e seus efeitos em crianças considerou o número de visitas às unidades de emergência médica, encontrando associação positiva para todos os poluentes, sendo a mais forte para $\mathrm{PM}_{10}$ e a mais fraca para $\mathrm{O}_{3}$. Constatou-se um aumento de $20 \%$ no número de crianças que procuraram o serviço de emergência com problemas respiratórios nos dias mais poluídos ${ }^{6}$.

Outro estudo demonstrou que a exposição materna a $\mathrm{CO}$ e $\mathrm{PM}_{10}$, durante o primeiro trimestre da gravidez, contribui para a diminuição do peso ao nascer e que um aumento da ordem de $1 \mathrm{ppm}$ de CO pode levar a um decréscimo de $23 \mathrm{~g}$ no peso ao nascer ${ }^{22}$. Estudo similar encontrou associação entre baixo peso ao nascer e a exposição materna no primeiro trimestre de gravidez a $\mathrm{CO}, \mathrm{NO}_{2}$ e $\mathrm{PM}_{10}{ }^{23}$.

Estudo específico sobre a poluição relacionada ao tráfego comparou a função pulmonar de um grupo de motoboys e taxistas à de um grupo controle e observou que os motoboys apresentaram a pior função pulmonar, o que pode estar associado à maior exposição aos poluentes veiculares ${ }^{24}$. Outro estudo comparou a pressão sanguínea e o eletrocardiograma de controladores de tráfego com os níveis de $\mathrm{CO}, \mathrm{PM}_{10}, \mathrm{NO}_{2}$ e $\mathrm{SO}_{2}$ e constatou que o aumento de $1,1 \mathrm{ppm}$ de CO pode levar a um aumento de $2,4 \mathrm{mmHg}$, na média, das pressões médias sistólicas e diastólicas ${ }^{25}$.

Um estudo sobre a correlação entre os níveis de $\mathrm{CO}, \mathrm{O}_{3}, \mathrm{SO}_{2}, \mathrm{NO}_{2}$ e $\mathrm{PM}_{10}$ e problemas respiratórios em idosos, antes e após a implementação do programa de restrição de circulação de veículos na região central de São Paulo, denominado "rodízio", verificou que este reduziu a concentração dos poluentes sem, entretanto, influenciar positivamente a saúde dos idosos ${ }^{26}$.
Dois trabalhos apontam que o impacto econômico devido aos efeitos à saúde decorrentes da exposição à poluição atmosférica no município de São Paulo é muito significativo e que estes efeitos e seus consequentes custos poderiam ser evitados ${ }^{27,28}$.

Os estudos analisados evidenciam o impacto significativo da poluição do ar na saúde da população do município de São Paulo, em idosos e crianças. As associações mais fortes foram encontradas entre os poluentes $\mathrm{SO}_{2}, \mathrm{PM}_{10}$ e CO, especialmente, para doenças respiratórias.

Embora os trabalhos tenham observado as correlações entre alguns poluentes e desfechos de saúde (como mortalidade e internação), não é possível avaliar o efeito isolado de cada poluente. Os estudos analisados trabalharam com períodos, populações e condições diferentes, dificultando a avaliação integrada das consequências causadas pelos poluentes na saúde da população da cidade de São Paulo.

A quase totalidade dos estudos utiliza para a caracterização da exposição à poluição do ar os dados das estações de monitoramento da qualidade do ar. Estes dados são dispersos e não evidenciam gradientes de exposição na região intraurbana associados, em especial, às vias de maior tráfego.

Como exemplo, é possível citar as estações de monitoramento de Cerqueira César e Congonhas que possuem representatividade de microescala (poucos metros no entorno da estação) $)^{29,30}$. A estação de Cerqueria César está localizada em situação topograficamente privilegiada, facilitando a dispersão de poluentes e fazendo com que a população possa estar exposta a níveis maiores de poluição que aqueles medidos por esta estação ${ }^{30}$. Ainda a estação de Congonhas, por estar situada entre vias de tráfego com alto volume de veículos, pode medir níveis de poluição maiores que aqueles aos quais a população está sujeita ${ }^{29}$. A estação de monitoramento do Ibirapuera parece ser a que mais representa a situação média de exposição e a poluição de background $^{31}$. Embora a utilização de média dos 
valores de poluição para representar a exposição da população não seja adequada, estes ainda são um dos únicos dados de medida sistemática possíveis de ser utilizados.

Deve ser destacado, ainda, que os estudos utilizam dados de internação hospitalar oriundos do Departamento de Informática do SUS (DATASUS), não incluindo as internações da rede privada, o que introduz vieses socioeconômicos nas análises.

Também deve ser destacado que os estudos de séries temporais são pouco efetivos para uma avaliação rápida de cenários e ou da eficiência de medidas específicas de controle da poluição do ar, uma vez que demandam períodos mais prolongados de observação.

\section{Avaliação da exposição à poluição relacio- nada ao tráfego}

Foram encontrados 122 artigos no Pubmed e utilizados 29 deles nesta revisão. Além dos artigos não relacionados à poluição veicular, alguns artigos sobre aplicação dos métodos em situações específicas foram descartados, tendo sido priorizadas apenas as aplicações mais recentes. A complexidade dos ambientes urbanos, o aumento da contribuição das fontes móveis e a diversidade de poluentes e dos efeitos à saúde da população têm impulsionado o desenvolvimento de métodos para a avaliação da exposição humana à poluição relacionada ao tráfego em áreas urbanas.

São seis os métodos principais de avaliação de exposição humana aos poluentes de fontes veiculares: 1) avaliação baseada na proximidade das vias; 2) interpolação estatística; 3) modelos de análise de uso do solo; 4) modelos de dispersão; 5) modelos integrados de emissão e meteorologia e 6) modelos híbridos - que combinam o monitoramento pessoal com um dos métodos anteriores $^{32}$.

Estudos baseados na proximidade consideram que quanto mais próximo da fonte de poluição, maiores serão as concentrações dos poluentes e, portanto, maior a exposição, ou seja, a distância entre as pessoas e a via é tomada como uma proxy da exposição aos poluentes relacionados ao tráfego. Estes estudos são úteis para avaliar o decaimento de poluentes e de seus efeitos em função da distância da via e também em análises exploratórias. No entanto, são criticados por considerarem um número restrito de covariáveis, não diferenciarem os tipos de poluentes envolvidos e a influência das condições meteorológicas e topográficas. Ainda, em ambientes urbanos complexos, indivíduos mais próximos da fonte não necessariamente são os mais expostos ${ }^{32}$.

Um exemplo deste método é o estudo que avaliou a relação entre a exposição ao material particulado fino e o desenvolvimento de Alzheimer em mulheres entre 68 e 79 anos. A exposição foi estimada pela distância da residência até a rua movimentada mais próxima. A conclusão foi que a exposição crônica ao material particulado de fontes veiculares pode estar envolvida no desenvolvimento da doença de Alzheimer. Foi encontrado risco de 1,9 (com intervalo de confiança de $95 \%$ de $0,3-4,1$ ) para o tráfego e testes de cognição em mulheres com mais de 74 anos $^{33}$. Vários outros exemplos são encontrados ${ }^{34-41}$.

$\mathrm{Na}$ interpolação estatística aplicada à avaliação da exposição, os dados de concentrações dos poluentes obtidos em estações de monitoramento são interpolados por meio de técnicas de geoestatística. A vantagem deste método é o uso de dados reais de poluição e a possibilidade da realização de estudos de dose-resposta. No entanto, este método não considera a influência das condições meteorológicas e fatores específicos do terreno na dispersão dos poluentes e, ainda, pode envolver erros significativos nas áreas de borda. A qualidade dos resultados depende da existência de uma densa rede de monitoramento, ou da coleta primária de dados, o que envolve custos elevados ${ }^{32}$.

Um estudo avaliou a relação entre a poluição do ar e a prevalência de diabetes mellitus. Técnicas de informação geográfica foram utilizadas para atribuir valores individuais de $\mathrm{NO}_{2}$, baseados em uma 
rede de amostradores. O estudo encontrou associação entre a diabetes mellitus cujas razões de chance variaram de 1.025 a 1.029, com intervalo de confiança de $95 \%^{42}$. Outro exemplo de estudo foi desenvolvido na Polônia ${ }^{43}$.

A análise de uso do solo estima a exposição, a partir de padrões de emissão, das características do uso do solo e do tráfego da área. As vantagens deste método são a possibilidade de analisar áreas sem dados de monitoramento e o baixo custo. As desvantagens são a dificuldade de inserção de condições específicas de uma determinada área e o trabalho em escala pequena, e a extrapolação de dados para áreas com uso e ocupação ou topografia muito específicos ${ }^{32}$.

Este método foi utilizado para o cálculo das concentrações de hidrocarbonetos em um estudo da exposição à poluição do ar durante a gravidez e sua associação com o peso ao nascer ${ }^{44}$. Diversos outros estudos utilizaram este método ${ }^{45-49}$. Para o município de São Paulo seriam necessários estudos para determinação dos padrões de emissão em função do uso e ocupação do solo.

Os modelos de dispersão também são empregados para estimar a exposição. Neste caso, informações de emissão, dados meteorológicos e topográficos são necessários para estimar a concentração dos poluentes. Alguns modelos integram módulos meteorológicos e químicos na simulação da dinâmica atmosférica dos poluentes, possibilitando uma estimativa mais realista da dispersão dos poluentes, quando comparada ao modelo de dispersão simples.

Estes modelos apresentam como vantagem a consideração das variações da poluição no tempo e espaço para diferentes escalas, mas requerem dados reais de poluição para validação e, muitas vezes, o desencontro temporal dos dados de entrada pode levar a estimativas errôneas. O custo da obtenção dos dados de entrada do modelo pode ser bastante elevado. A necessidade de computadores de alto desempenho, softwares e pessoal treinado também pode ser um fator limitante para o uso destes modelos ${ }^{32}$.
O impacto da exposição aos poluentes veiculares foi avaliado na Áustria, França e Suíça. A utilização de modelos permitiu o cálculo da concentração de $\mathrm{PM}_{10}$ para uma grid de $1 \mathrm{~km}^{2} \mathrm{e}$, a partir desta estimativa, foi possível determinar a fração veicular. Os dados foram associados à mortalidade ( $\geq 30$ anos), hospitalização por causas cardiovasculares ou respiratórias, incidência de bronquite crônica ( $\geq 25$ anos), episódios de bronquite em crianças e ataques de asma. Os resultados mostraram que $6 \%$ da mortalidade da população foi devida à exposição a poluentes atmosféricos, sendo que $50 \%$ destas mortes foram por conta das emissões veiculares. O tráfego ainda foi responsável por 25 mil novos casos de bronquite crônica em adultos, mais de 290 mil casos de bronquite em crianças e 500 mil de ataques de asma ${ }^{50}$. Diversos outros estudos utilizaram métodos semelhantes $\mathrm{s}^{51-53}$.

Estudos deste tipo são viáveis para o município de São Paulo, sendo a maior dificuldade a qualidade dos dados disponíveis para a alimentação do modelo e sua vantagem é a possibilidade de análise, com alta resolução, de cenários envolvendo medidas de controle, como restrição do uso de veículos, mudança de combustíveis ou de perfil da frota.

Outros modelos híbridos de avaliação da exposição combinam o uso de monitoramento pessoal com um dos métodos citados anteriormente. Neste caso, o principal objetivo é validar resultados do monitoramento para outras escalas. Estes modelos permitem validar as medidas de concentrações ambientais de poluentes como parâmetro para avaliação da exposição humana. No entanto, o monitoramento pessoal é caro e depende da facilidade/custo da análise e sua aplicação requer que as demais técnicas de avaliação da exposição já tenham sido empregadas ${ }^{32}$.

Um exemplo é o estudo que combinou técnicas de interpolação de dados de monitoramento e modelos de uso e ocupação do solo, para avaliar a exposição à poluição do ar e correlacioná-la à mortalidade e incidência de câncer de pulmão. Para um aumento 
de $10 \mu \mathrm{g} / \mathrm{m}^{3}$ de fumaça preta, houve um risco relativo de 1,03 (0,88-1,2), com intervalo de confiança de $95 \%$ para mortalidade de câncer de pulmão ${ }^{54}$. Outros estudos usaram métodos combinados para avaliar a exposição ao tráfego ${ }^{55,56}$.

Outros métodos de avaliação da exposição aos poluentes veiculares, como por exemplo, o uso de questionários, em que são reportados os níveis de tráfego, monitores instalados em residências, amostradores pessoais ou passivos e dados de estação de monitoramentos também são aplicáveis $^{57-61}$.

\section{Conclusões}

Os estudos sobre os efeitos à saúde da população do município de São Paulo causados pela exposição à poluição do ar mostram que esta contribui para o aumento da mortalidade e morbidade, principalmente por doenças respiratórias e cardiovasculares nos grupos vulneráveis. A maioria dos trabalhos sobre efeitos à saúde considera valores médios da concentração de poluentes para o município, os quais não evidenciam gradientes de exposição, ou seja, áreas críticas de picos de poluição.

O município de São Paulo apresenta complexa distribuição de fontes móveis e estacionárias de poluição do ar. Além disso, a dispersão dos poluentes sofre influência de parâmetros atmosféricos e do uso do solo como, por exemplo, a presença de altos edifícios, que influenciam o deslocamento de ventos e a presença de ilhas de calor.

Diversas especificidades da exposição da população aos poluentes atmosféricos ainda precisam ser investigados. Por exemplo, a contribuição por tipo de veículo (diesel, gasolina, álcool e flex), por poluente para os desfechos de saúde e do longo tempo gasto em congestionamentos diários. Além disso, a coleta de informações sobre os atendimentos que não resultaram em internação deve ser priorizada.

Há uma diversidade de métodos de avaliação da exposição à poluição gerada pelo tráfego que podem ser utilizados isoladamente ou em conjunto. O desenvolvimento das técnicas de geoprocessamento também tem ampliado as possibilidades de abordagem.

As dificuldades investigativas levantadas não são exclusividade do Brasil, mas da maioria dos países em desenvolvimento, onde o principal desafio é a falta de dados adequados para avaliação da exposição. No entanto, isto não pode ser um impedimento, pelo contrário, deve ser uma motivação para a busca de soluções, especialmente nas grandes regiões metropolitanas. Diversas alternativas metodológicas podem ser empregadas isoladamente ou de forma complementar.

\section{Referências}

1. Ribeiro H, Assunção JV. Historical overview of air pollution in São Paulo metropolitan area, Brazil: influence of mobile sources and related health effects. In: Sucharov LJ, Brebbia CA. (editors). Urban transport and the environment in the $21^{\text {st }}$ century. Boston: Wit Press; 2001. p 351-360.

2. Lacasaña-Navarro M, Aguilar-Garduño C, Romieu I. Evolucion de la contaminacion del aire e impacto de los programas de control em tres megaciudades de America Latina. Salud Pública Méx. 1999;41(3):203-15.

3. Companhia de Tecnologia de Saneamento Ambiental. Relatório de Qualidade do Ar no Estado de São Paulo 2008 [internet]. São Paulo: CETESB; 2009. p 340. [citado 24 set 2009]. Disponível em: <http://www.cetesb.sp.gov.br/ar/ publicacoes.asp $>$.
4. Instituto Brasileiro de Geografia e Estatística (IBGE). [site na internet]. [citado 29 nov 2009]. Disponível em: <www.ibge. gov.br>.

5. Prefeitura Municipal da Cidade de São Paulo (PMSP). [citado 2009 Bov 29]. Disponível em: www.prefeitura. sp.gov.br.

6. Lin CA, Martins MA, Farhat SCL, Pope IIICA, Conceição GMS, Anastácio VM, et al. Air pollution and respiratory illness of children in São Paulo, brazil. Paediatr Perinatal Epidemiology. 1999;13(4):475-88.

7. Gouveia N, Fletcher T. Time series analysis of air pollution and mortality: effects by cause, age and socioeconomic status. J Epidemiol Community Health. 2000;54(10):750-5. 
8. Braga, ALF, Saldiva PH, Pereira LA, Menezes JJ, Conceição GM, Lin CA, et al. Health effects of air pollution exposure on children and adolescents in São Paulo. Brazil. Pediatr Pulmonol. 2001;31(2):106-13.

9. Martins LC, Latorre DO, Cardoso MRA, Gonçalves FLT, Saldiva PHN, Braga ALF. Poluição atmosférica e atendimentos por pneumonia e gripe em São Paulo, Brasil. Rev Saúde Pública. 2002;36(1):88-94.

10. Botter DA, Jorgensen B, Peres AAQ. A longitudinal study on mortality and air pollution for São Paulo, Brazil. Journal Expo Anal Environ Epidemiol. 2002;12(5):335-43.

11. Martins MCH, Fatigati FJ, Véspoli TC, Martins LC, Pereira LAA, Martins MA, et al. Influence of socioeconomic conditions on air pollution adverse health effects in elderly people: an analysis of six regions in São Paulo, Brazil. J Epidemiol Community Health. 2004;58(1):41-6.

12. Gouveia N,. Mendonça GA, Ponce de Leon A, Correia JEM, Junger WL, Freitas CU, et al. Poluição do ar e efeitos na saúde nas populações de duas grandes metrópoles brasileiras. Epidemiol Serv Saúde. 2003;12(1): 29-40.

13. Conceição GMS, Miraglia SGEK, Kishi HS, Saldiva PHN, Singer JM. Air pollution and child mortality: a time-series study in São Paulo, Brazil. Environ Health Perspect. 2001;109 (Suppl 3):347-50.

14. O'Neill M.S. Bell ML, Ranjit N, Cifuentes LA, Loomis D, Gouveia N, et al. Air pollution and mortality in Latin America: the role of education. Epidemiology. 2008;19(6):810-9.

15. Pereira LA, Loomis D, Conceição GM, Braga AL, Arcas RM, Kishi HS, et al. Association between air pollution and intrauterine mortality in Sao Paulo, Brazil. Environ Health Perspect. 1998;106(6):325-9.

16. Gouveia N, Bremner SA, Novaes HMD. Association between ambient air pollution and birth weight in São Paulo, Brazil. J Epidemiol Community Health. 2004;58(1):11-7.

17. Braga ALF, Conceição GMS, Pereira LAA, Kishi HS, Pereira JCR, Andrade MF, et al. Air pollution and pediatric respiratory hospital admissions in São Paulo, Brazil. J Environ Medicine. 1999;1(2):95-102.

18. Gouveia N, Fletcher T. Respiratory diseases in children and outdoor air pollution in São Paulo, Brazil: a time series analysis. Occup Environ Med. 2000;57(7):477-83.

19. Ribeiro H, Cardoso MRA. Air pollution and children`s health in São Paulo (1986-1998). Soc Sci Med. 2003;57(11):2013-22.

20. Freitas C, Bremner S., Gouveia N, Pereira LAA, Saldiva PHN. Internações e óbitos e sua relação com a poluição atmosférica em São Paulo, 1993 a 1997. Rev Saúde Pública. 2004;38(6):751-7.

21. Lin CA, Pereira LAA, Conceição GMSC, Kishi HS, Milani Junior R, Braga ALF, et al. Association between air pollution and ischemic cardiovascular emergency room visits. Environ Res. 2003(1);92:57-63.

22. Santos UP, Terra-Filho M, Lin CA, Pereira LA, Vieira TC, Saldiva PH, Braga AL. Cardiac arrhythmia emergency room visits and environmental air pollution in São Paulo, Brazil. J Epidemiol Community Health. 2008;62(3):267-72.

23. Medeiros A, Gouveia N. Relação entre baixo peso ao nascer e a poluição do ar no Município de São Paulo. Rev Saúde Pública. 2005;39(6):965-72.

24. Ferreira CAS, Santos FL, Costa IP, Pereira LV, Rego PBL. Análise da função pulmonar de motoboys: o efeito da poluição atmosférica. Prevenção, a melhor forma de cuidar. Mundo Saúde (Impr). 2009;32(2):170-4.

25. Santos UP, Braga AL, Giorgi DM, Pereira LA, Grupi CJ, Lin CA. Effects of air pollution on blood pressure and heart rate variability: a panel study of vehicular traffic controllers in the city of São Paulo, Brazil. Eur Heart J. 2005(2);26:193-200.

26. Martins LC, Latorre MRDO, Saldiva PHN, Braga ALF. Relação entre poluição atmosférica e atendimentos por infecção de vias aéreas superiores no município de São Paulo: avaliação do rodízio de veículos. Rev Bras Epidemiol. 2001;4(3):220-9.

27. Bell ML, Davis DL, Gouveia N, Borja-Aburto VH, Cifuentes LA. The avoidable health effects of air pollution in three Latin American cities: Santiago, São Paulo and Mexico city. Environ Res. 2006;100(3):431-40.

28. Miraglia SG, Saldiva PH, Böhm GM. An evaluation of air pollution health impacts and costs in São Paulo, Brazil. Environ Manage. 2005;35(5):667-76.

29. Companhia de Tecnologia de Saneamento Ambiental. Caracterização das estações da rede de monitoramento da qualidade do ar na RMSP - estação Congonhas [internet]. São Paulo: CETESB; 2004. p 64. [citado 7 fev 2010]. Disponível em: <http://www.cetesb.sp.gov.br/ar/qualidade-do-ar/31publicacoes-e-relatorios $>$.

30. Companhia de Tecnologia de Saneamento Ambiental. Caracterização das estações da rede de monitoramento da qualidade do ar na RMSP - estação Cerqueira César [internet]. São Paulo: CETESB; 2004. p 51. [citado $10 \mathrm{fev}$ 2010]. Disponível em: <http://www.cetesb.sp.gov.br/ar/ qualidade-do-ar/31-publicacoes-e-relatorios $>$.

31. Companhia de Tecnologia de Saneamento Ambiental. Caracterização das estações da rede de monitoramento da qualidade do ar na RMSP - estação Ibirapuera [internet]. São Paulo: CETESB; 2004. p 66. [citado 7 fev 2010]. Disponível em: <http://www.cetesb.sp.gov.br/ar/qualidade-do-ar/31publicacoes-e-relatorios $>$.

32. Jerrett M Arain A, Kanaroglou P, Beckerman B, Potoglou D, Sahsuvaroglu T, et al. A review and evaluation of intraurban air pollution exposure models. J Expo Anal Environ Epidemiol. 2005;15(2):185-204.

33. Ranft U, Schikowski T, Sugiri D, Krutmann J, Krämer U, Long-term exposure to traffic-related particulate matter impairs cognitive function in the elderly. Environ Res. 2009;109(8):1004-11.

34. de Medeiros .AP, Gouveia N, Machado RP, de Souza MR, Alencar GP, Novaes HM, et al. Traffic-related air pollution and perinatal mortality: a case-control study. Environ Health Perspect. 2009;117(1):127-32. 
35. Williams LA, Ulrich CM, Larson T, Wener MH, Wood B, Campbell PT, et al. Proximity to traffic, inflammation, and immune function among women in the Seatle, Washington, area. Environ Health Perspect. 2009;117(3):373-8.

36. Chang J, Delfino RJ, Gillen D, Tjoa T, Nickerson B, Cooper D. Repeated respiratory hospital encounters among children with asthma and residential proximity to traffic. Occup Environ Med. 2009;66(2):90-8.

37. Medina-Ramón M, Goldberg R, Melly S, Mittleman MA, Schwartz J. Residential exposure to traffic-related air pollution and survival after heart failure. Environl Health Perspect. 2008;116(4):481-5.

38. Morgenstern V, Zutavern A, Cyrys J, Brockow I, Koletzko S, Krämer U, et al. Atopic deseases, allergic sensitization, and exposure to traffic-related air pollution in children. Am J Respir Crit Care Med. 2008;177(12):133-7.

39. Shima M, Nitta Y, Adachi M. Traffic-related air pollution and respiratory symptoms in children living along trunk roads in Chiba Prefecture, Japan. J Epidemiol. 2003;13(2):108-19.

40. Wilhelm M, Ritz B. Residential proximity to traffic and adverse birth outcomes in Los Angeles county, California, 1994-1996. Environ Health Perspect. 2003;112(2):207-16.

41. Hoek G, Brunekreef B, Goldbohm S, Fischer P, van den Brandt. Association between mortality and indicators of traffic-related air pollution in Netherlands: a cohort study. Lancet.2002;360(19):1203-9.

42. Brook RD, Jerret M, Brook JR, Bard RL, Finkelstein MM. The relationship between diabetes mellitus and traffic-related air pollution. J Occup Environ Med. 2008;50(1):32-8.

43. Liebhart J, Malolepszy J, Wojtyniak B, Pisiewicz K, Plusa T, Gladysz U. Prevalence and risk factors for asthma in Poland: results from the PMSEAD study. J Investig Allergol Clin Immunol. 2007;17(6):367-74.

44. Aguilera I, Guxens M, Garcia-Esteban R, Corbella T, Nieuwenhuijsen MJ, Foradada CM, et al. Association between GIS-based exposure to urban air pollution during pregnancy and birth weight in the INMA Sabadell Cohort. Environ Health Perspect. 2009;117(8):1322-7.

45. Su JG, Jerrett M, Beckerman B, Wilhelm M, Ghosh JK, Ritz B. Predicting traffic-related air pollution in Los Angeles using a distance decay regression selection strategy. Environ Res. 2009;109(6):657-70.

46. Jerret M, Finkelstein MM, Brook JR, Arain MA, Kanaroglou P, Stieb DM, et al. A cohort study of traffic-related air pollution and mortality in Toronto, Ontario, Canada. Environ Health Perspect. 2009;117(5):772-7.

47. von Klot S, Gryparis A, Tonne C, Yanosky J, Coull BA, Goldberg RJ et al. Elemental carbon exposure at residence and survival after acute miocardial infarction. Epidemiology. 2009;20(4):547-54.

48. Poplawski K, Poplawski K, Gould T, Setton E, Allen R, $\mathrm{Su}$ J, Larson $\mathrm{T}$ et al. Intercity transferability of land use regression models for estimating ambient concentrations of nitrogen dioxide. J Exp Sci Environ Epidemiol. 2008;19(1):107-17.
49. Rosenlund M, Picciotto S, Forastiere F, Stafoggia M, Perucci CA. Traffic-related air pollution in relation to incidence and prognosis of coronary heart disease. Epidemiology. 2008;19(1):121-8.

50. Künzli N, Kaiser R, Medina S, Studnicka M, Chanel O, Filliger P, et al. Public-health impact of outdoor and trafficrelated air pollution: an European assessment. Lancet. 2000;356(9232):795-801.

51. Lindgren A, Stroh E, Nihlén U, Montnémery P, Axmon A, Jakobsson K, et al. Traffic exposure associated with allergic asthma and allergic rhinitis in adults. A cross-sectional study in southern Sweden. Int J Health Geogr. 2009;8(25).

52. Delfino RJ, Chang J, Wu J, Ren C, Tjoa T, Nickerson B, et al. Repeated hospital encounters for asthma in children and exposure to traffic-related air pollution near the home. Ann Allergy Asthma Immunol. 2009;102(2):138-44.

53. Nordling E, Berglind N, Melén E, Emenius G, Hallberg J, Nyberg F, et al. Traffic-related air pollution and childhood respiratory symptoms, function and allergies. Epidemiology. 2008;19(3):401-8.

54. Brunekreef B, Beelen R, Hoek G, Schouten L, Bausch-Goldbohm $\mathrm{S}$, Fischer P, et al. Effects of long-term exposure to traffic-related air pollution on respiratory and cardiovascular mortality in the Netherlands: the NLCS-AIR study. Res Rep Health Eff Inst. 2009;139:5-71.

55. Rosenlund M, Forastiere F, Porta D, De Sario M, Badaloni C, Perucci CA. Traffic-related air pollution in relation to respiratory symptoms, allergic sensitization and lung function in schoolchildren. Thorax. 2009;64(7):573-80.

56. Van Roosbroeck S, Li R, Hoek G, Lebret E, Brunekreef B, Spiegelman D. Traffic-related outdoor air pollution and respiratory symptoms in children: the impact of adjustment for exposure measurements error. Epidemiology. 2008;19(3):409-16.

57. Berglind N, Bellander T, Forastiere F, von Klot S, Aalto P, Elosua R, et al. Ambient air pollution and daily mortality among survivors of myocardial infarction. Epidemiology. 2009;20(1):110-8.

58. Fan ZT, Meng Q, Weisel C, Laumbach R, Ohman-Strickland P, Shalat $\mathrm{S}$, et al. Acute exposure to elevated $\mathrm{pm}_{2.5}$ generated by traffic and cardiopulmonary health effects in health older adults. J Exp Sci Environ Epidemiol. 2009;19(5):525-33.

59. Escamilla-Nuñez MC, Barraza-Villarreal A, Hernandez-Cadena L, Moreno-Macias H, Ramirez-Aguilar M, Sienra-Monge JJ, et al. Traffic-related air pollution and respiratory symptoms among asthmatic children, resident in Mexico City: the EVA cohort study. Respir Res. 2008;9(1):74-85.

60. Jerret M, Shankardass K, Berhane K, Gauderman WJ, Künzli N, Avol E, et al. Traffic-related air pollution and asthma onset in children: a prospective cohort study with individual exposure measurement. Environ Health Perspect. 2008;116(10):1433-8.

61. Lee YL, Su HJ, Sheu HM, Yu HS, Guo YL. Traffic-related air pollution, climate, and prevalence of eczema in Taiwanese school children. J Invest Dermatol. 2008;128(10): 2412-20.

Recebido em: 17/08/2010 Versão final apresentada em: 30/03/2011 Aprovado em: 06/06/2011 